\title{
Paraffin Sections of Sputum Block: Description of a New Method for Pathological and Molecular Study
}

\author{
Arshad Rahmani ${ }^{1,283^{*}}$, Mohammad Alzohairy ${ }^{1}$, Habeeb Khadri ${ }^{1}$, \\ Moshahid A. Rizvi ${ }^{2}$ and Ashish Mandal ${ }^{3}$ \\ ${ }^{1}$ Department of Medical Laboratory Science, College of Applied Medical Sciences, \\ Qassim University, Kingdom of Saudi Arabia. \\ ${ }^{2}$ Department of Biosciences, Jamia Millia Islamia, New Delhi, India. \\ ${ }^{3}$ Department of Pathology, Maulana Azad Medical College, New Delhi, India.
}

Short Communication

Received $9^{\text {th }}$ June 2011

Accepted $11^{\text {th }}$ June 2011

Online Ready $13^{\text {th }}$ June 2011

\section{ABSTRACT}

Aim: This study was designed to enhance the scope of sputum analysis by using it as a clinical tool in gene/protein expression, by making the paraffin embedded blocks.

Methodology: The specimens were prepared as smear and cell blocks for cytopathologic examination. The preparation of paraffin-embedded block from sputum samples employs fixation and dehydration of the cell specimens. The sputum specimen is first exposed to a suitable fixating agent, Formalin, and graded acetone was employed to dehydrate the samples and saved as pellet. Paraffin blocks containing embedded pellet were taken out gently from the tube. The solidified paraffin-embedded tissue blocks through this novel approach were found to be easy to process for immunohistochemistry and molecular analysis.

Results: Immunohistochemistry staining was performed on paraffin section of sputum block for Cytokeratin (CK) and was found to be easy to process for immunohistochemistry.

Conclusion: The sputum block preparation is feasible and non-invasive, can be useful to identify new biomarkers of exposure or susceptibility in patients with lung pathology to enhance the understanding of airways changes due to different etiological factors and may be useful to find new biomarkers in order to assess and monitor early lung damage.

Keywords: Sputum block; immunohistochemistry; molecular; cytokeratin; 


\section{INTRODUCTION}

Sputum examination is one of the most commonly prescribed preliminary investigations in evaluating the patients of respiratory diseases. Sputum analysis has several advantages over other techniques for being non-invasive and easily applicable on a large scale in followup studies. It is used to study various biological markers for diseases like any form of inflammation in airway diseases, COPD, asthma, lung cancer, cystic fibrosis. Therefore sputum examination is employed in almost all cases of respiratory dysfunctions involving the tracheo-broncheal tree, however further cytological examination of material after bronchoscopy or lung biopsy becomes essential in inconclusive cases to correctly diagnose and type carcinomas or any other lung pathologies. This study therefore was designed to enhance the scope of sputum analysis by using it as a clinical tool in gene/protein expression, by making the paraffin embedded blocks.

The nature and extent of pulmonary diseases has, until recently, been assessed by direct invasive bronchoscopy with bronchial washings, biopsy, bronchoalveolar lavage, or by indirect measurements of symptoms, pulmonary function tests or peripheral blood inflammatory markers (Fireman, 2003). Sputum examination provides specific information on both cellular and molecular constituents in airway inflammation. It was first used to investigate lung cancer and respiratory infections (Bickerman, et al., 1958; Yue and Cohen, 1967) and later to diagnose Pneumocystis carinii pneumonia in patients infected with HIV (Leigh et al., 1989). More recently, the method was modified to study patients of asthma by focusing on several markers of inflammation as well as for investigating the pathogenesis, pathophysiology and treatment of asthma in general and of occupational asthma as well (Malerba et al., 2008). New non-invasive techniques eNO, induced sputum and exhaled breath condensate have been successfully introduced to study the inflammation processes involved in the pathogenesis of pulmonary diseases while other methods to directly assess airway inflammation that have been tried before are quite invasive (Barak et al., 2004).

Cytokeratins, released from the epithelial lining of tracheobronchial tree in the sputum may provide useful markers for epithelial malignancies, distinctly reflecting ongoing cell activity, upon release from proliferating or apoptotic cells. It appears that motifs in certain cytokeratins make them likely substrates for caspase degradation, and their subsequent release occurs during the intermediate events in apoptosis. The standardization of IHC on sputum blocks is an important tool that involves technical procedures i.e. antigen retrieval and interpretation. Sputum block preparation was used in an attempt to standardize IHC on sputum blocks and was successfully done. Most sensitive part of this procedure is fixation of samples and processing of the paraffin-embedded sputum blocks.

The demand for preparation of paraffin-embedded blocks from sputum for Immunohistochemistry is ever more pressing because of the emergence of a new field of translational research that requires of the differential expression of various prognostic markers.

\section{MATERIALS AND METHODS}

Sputum samples from the textile stitching industry workers were collected in a wide mouth glass jar after chest percussion. Formalin (10\%) was added to the sample; within 2 hours of collection (effect of formalin on tissue antigenicity is dependent on the time of fixation) (Fox et al., 1985) and which was then centrifuged at $1000 \mathrm{rpm}$ for 10 minutes and pellet saved. It was then subjected to graded acetone ( $30 \%$ to absolute) to dehydrate the samples. Picric 
acid (1\%) was added to the pellet (contact period 3-5 minutes), which stained the pellet yellow. The pellet was dried in an oven at $46^{\circ} \mathrm{C}$ for an hour. Molten paraffin wax $\left(56^{\circ} \mathrm{C}\right)$ was added in the tube containing the pellet and left for about three hours. Paraffin blocks containing embedded pellet were taken out gently from the tube by tapping it and were used for sectioning. Five micron thick sections of the sputum block were cut \& taken on the poly Ilysine coated glass slides.

An immunohistochemical staining for Cytokeratin was performed on consecutive paraffin sections using streptavidin-biotin method (Stephen et al., 2003). Antigen retrieval was done by microwave treatment (Shi Shan-Rong, et al., 2002). Then slides were incubated with primary antibody followed by secondary biotinylated antibody (for 20 minutes). After washing, sections were incubated with streptavidin peroxidase for 20 minutes. Finally the enzyme was visualized after 2 to 3 minutes of incubation with the Diaminobenzedine (DAB).

Sputum smears on poly I-lysine coated slide were also made for immunohistochemical staining. DNA extraction was also done in these paraffin sections of sputum using (Anonymous, 2011). Sputum smears on poly I-lysine coated slide were also made for immunohistochemical Staining (Shi Shan-Rong et al., 2002).

\section{RESULTS AND DISCUSSION}

Five micron thick sections of the sputum block were cut \& taken on the poly l-lysine coated slides. Immunohistochemistry staining was performed on paraffin section of sputum block for Cytokeratin (CK) and was found to be easy to process for immunohistochemistry (Figure 1).

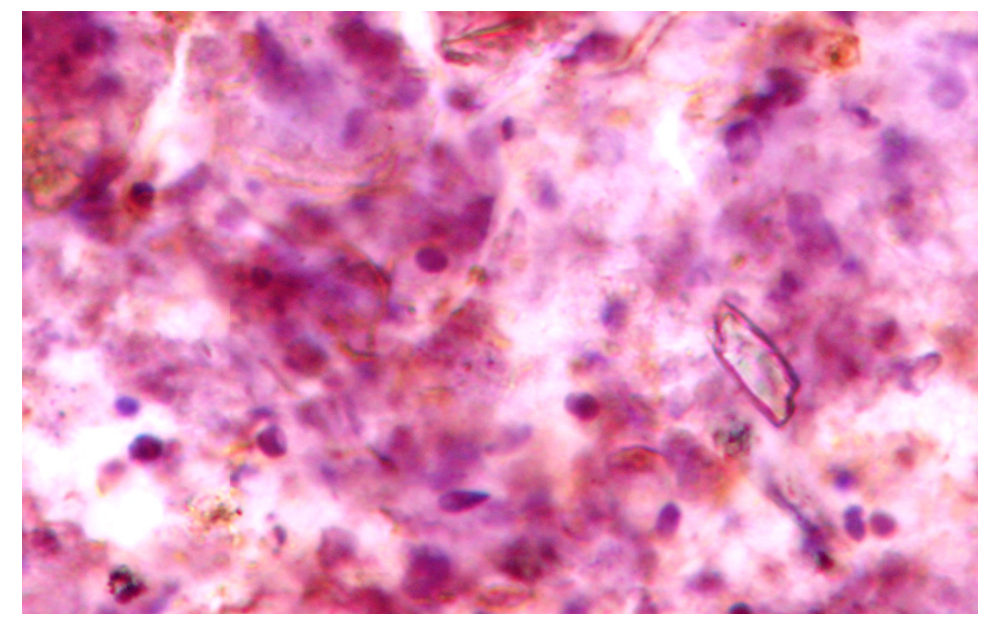

Fig. 1. Cytokeratin expression in sputum blocks section

Sputum smears which were subjected to immunohistochemical staining using the antigen retrieval method did not show any result as the smear got washed off during retrieval.

DNA extraction was also done and the amount of DNA yield was measured and was found to be (40 ng /ul) sufficient for analysis. 
Our novel approach in the study paraffin-embedded block from sputum through the histological section or the slide is likely to open new vistas in the field of Immunohistochemistry and cytology. Many different kinds of studies like protein expression, cellular damage, apoptosis or other molecular analysis can be done on paraffin-embedded block from sputum with greater ease and authenticity.

We have done immunohistochemistry Assay and DNA extraction on paraffin sections of sputum block. Our results revealed that the preparation of sputum blocks is reliable, simple method for detection of protein expression on routinely formalin-fixed, paraffin-embedded sections in daily pathological diagnosis. DNA extraction was also done in these paraffin sections of sputum. The DNA yields was evaluated by spectrophotometry and was found to be sufficient (40 $\mathrm{ng} / \mathrm{ul})$ or analysis.

Sputum blocks technique is still at the developmental stages, it can be widely used in immunohistochemical studies especially where sputum smear analysis is difficult.

Application of standardized protocols of sputum blocks on IHC may lead to valuable retrospective studies in various fields of pathology involving tracheobronchial tree. Further studies may examine the practical use of sputum blocks in diagnostic pathology.

\section{CONCLUSION}

The sputum block preparation is feasible and non-invasive, can be useful to identify new biomarkers of exposure or susceptibility in patients with lung pathology to enhance the understanding of airways changes due to different etiological factors and may be useful to find new biomarkers in order to assess and monitor early lung damage initiated by inducers in order to prevent the progression of lung disease.

\section{REFERENCES}

Bickerman, H.A., Sproud, E.E., Barach, A.L., et al. (1958). An aerosol method of producing bronchial secretions in human subjects: a clinical technique for detection of lung cancer. Dis. Chest, 4, 347-362.

Barak, V., Helena, G., Katja, W., et al. (2004). Clinical utility of cytokeratins as tumor markers. Clin. Biochem., 37(7), 529-540.

Fireman, Elizabeth. (2003). Induced Sputum as a Diagnostic Tactic in Pulmonary Diseases. IMAJ, 5, 524-527.

Fox, C.H., Johnson, F.B., Whiting, J., et al. (1985). Formaldehyde fixation. J. Histochem. Cytochem., 33, 845-853.

Anonymous. (2011). DNA Extraction from paraffin embedded block by DNA Extraction Qiagen kit. Available from: http://research.amnh.org/genomics/f/lab\%20protocol.pdf.

Shi Shan-Rong, Richard, J.C., Lin, W., et al. (2002). DNA Extraction from Archival formalinfixed, Paraffin-embedded Tissue Sections Based on the Antigen Retrieval Principle: Heating under the Influence of pH. J. Histochem. Cytochem., 50, 1005-1011.

Leigh, T.R., Hume, C., Gazzard, B., et al. (1989). Sputum induction for diagnosis of Pneumocystis carinii pneumonia. Lancet, 205-206.

Lemiere, C., Pizzichini, M.M., Balkissoon, R., et al. (1999). Diagnosing occupational asthma: use of induced sputum. Eur. Respir. J., 13, 482-488. 
Malerba, M.B., Ragnoli, M. Corradi. (2008). Non-invasive methods to assess biomarkers of exposure and early stage of pulmonary disease in smoking subjects. Monaldi Arch. Chest Dis., 69, 3, 128-133.

Stephen, P. Slone, Donald, R. Fleming, John, J. Buchino. (2003). Sinus Histiocytosis With Massive Lymphadenopathy and Langerhans Cell Histiocytosis Express the Cellular Adhesion Molecule CD31. Arch. Pathol. Lab. Med., 127, 341-344.

Yue, W.Y., Cohen, S.S. (1967). Sputum induction by newer inhalation methods in patients with pulmonary tuberculosis. Dis. Chest, 51(6), 614-20.

(C) 2011 Rahmani et al.; This is an Open Access article distributed under the terms of the Creative Commons Attribution License (http://creativecommons.org/licenses/by/3.0), which permits unrestricted use, distribution, and reproduction in any medium, provided the original work is properly cited. 